Revista de Psicología y Educación / Journal of Psychology and Education, 2020, 15(2), 166-183 (www.rpye.es) Doi: https://doi.org/10.23923/rpye2020.02.194

ISSN: $1699-9517$

\title{
Clima familiar y su relación con el rendimiento académico en estudiantes de Bachillerato
}

\author{
Javier Álvarez-Bermúdez y Francisco Javier Barreto-Trujillo* \\ Universidad Autónoma de Nuevo León
}

\begin{abstract}
Resumen: El objetivo del presente estudio fue indagar acerca de la relación entre el clima familiar y el rendimiento académico en estudiantes de preparatorias públicas del estado de Nuevo León, México. Los participantes fueron 290 adolescentes estudiantes de Bachillerato de ambos sexos (51\% varones y $49 \%$ mujeres), con edades comprendidas entre 15 a 22 años, $(M=16.36$; $D T=0.06)$. Se realizaron análisis de correlación de las variables de estudio, así como ANOVA contrastando el clima familiar y el promedio general para observar probables diferencias entre los grupos analizados. Los resultados mostraron que la dimensión que se relaciona de forma más significativa con el rendimiento académico es la interacción familiar negativa. Igualmente se encontraron diferencias significativas entre las relaciones familiares negativas y el bajo rendimiento académico. Finalmente, se discuten los resultados y sus posibles implicaciones prácticas. Palabras clave: Clima familiar, Rendimiento académico, Bachillerato, Correlaciones, ANOVA.
\end{abstract}

Family climate and its relation to academic performance in high school students

\begin{abstract}
The objective of the present study was to investigate the relationship between family climate and academic performance in public high school students in the state of Nuevo León, Mexico. The participants were 290 adolescent high school students of both sexes (51\% male and $49 \%$ female), aged between 15 and 22 years, $(M=16.36, S D=0.064)$. Correlation analyses of the study variables were performed, as well as ANOVA contrasting the family climate and the general mean to observe possible differences between the analyzed groups. The results showed that negative family interaction is the dimension most significantly related to academic performance. Likewise, significant differences were found between negative family relationships and low academic performance. Finally, the results and their possible practical implications are discussed.

Keywords: Family climate, Academic performance, High school, Correlations, ANOVA.
\end{abstract}

La familia es el primer grupo de referencia que tenemos las personas y donde se desarrollan los primeros conocimientos e interacciones con la sociedad. Asimismo ahí se establecen los roles a seguir en las relaciones sociales futuras. La familia es la unión de personas que desarrollan sentimientos de pertenencia través de sus relaciones y establecen vínculos de reciprocidad e independencia entre los integrantes de este grupo social (Mazo,

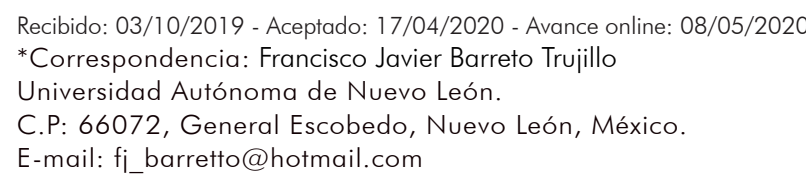

Álvarez-Bermúdez, J. y Barreto-Trujillo, F. J. (2020). Clima familiar y su relación con et rendimiento académico en estudiantes de Bachillerato. Revista de Psicología y Educación rendimiento académico en estudiantes de Bachillerato. Revista
15(2), 166-183, https://doi.org/10.23923/rpye2020.02.194
Mejía, y Muñoz, 2019). Además, construyen compromisos interpersonales entre sus miembros y comparten un proyecto de vida. En ese sentido, la familia tiene como parte de sus funciones desarrollar en los hijos las herramientas o competencias que les permitan hacer frente a las exigencias que los diversos entornos sociales les presentarán (Olson et al., 1989). Como grupo social, la familia transmite las creencias, los valores, normas, formas de afrontar los problemas que se les plantean y los comportamientos esperados en la sociedad en la cual se desarrolla, de ahí que las interacciones que se dan entre los miembros de la familia se convierten en 1699-9517/@ 2020 Asociación Científica de Psicología y Educación (ACIPE). Publicado por Consejo General de Colegios Oficiales de Psicólogos, España. Este es un artículo Open Access bajo la CC BY-NC-ND licencia (http://creativecommons.org/licencias/bync-nd/4.0/). 
un indicador endogrupal de cómo serán las relaciones de sus miembros con las personas e instituciones de su sociedad (Oliva y Villa, 2014). Se considera que los conceptos de cohesión, adaptabilidad y comunicación como características de la familia serán relevantes para entender cómo funciona la misma (Martínez, Merino, Iriarte y Olson, 2017).

Dentro de la familia se desarrolla el clima familiar, entendido esto como la forma en como los integrantes de dicha familia la perciben y actúan dentro de la misma (Estévez, Murgui, Musitu y Moreno, 2008). El clima familiar tiene una connotación afectiva y educativa de gran importancia, el clima familiar permite el proceso de desarrollo de las habilidades sociales en los hijos por medio de diversos mecanismos y estrategias, donde juega un papel esencial el ambiente que se desarrolla en el núcleo familiar (Moos, Moss y Tricket, 1984). Las interacciones que los padres desarrollan con los hijos en el hogar pueden variar en cantidad y calidad. El clima familiar está constituido por el ambiente percibido e interpretado por los miembros que integran la familia, el cual ha mostrado ejercer una influencia significativaen el desarrollo social, físico, afectivo e intelectual, así como en la conducta de sus integrantes (Moreno, Estévez, Murgui y Musitu, 2009).

El clima familiar está relacionado con las interacciones de los padres y de los hijos en el hogar. Estas interacciones pueden variar en cantidad y calidad. Según el tipo de interacción familiar que se establece en las personas desde edades tempranas, ejercen influencia en las diferentes etapas de la vida. Cuando se presenta una relación conflictiva dificulta el desarrollo psicosocial del sujeto. Cuando es una relación positiva permite una madurez emocional y mejor desarrollo en los ámbitos sociales, escolares y laborales (Verdugo et al., 2014). El clima afectivo que se presenta en la familia es parte importante para el desarrollo de las personas. Un ambiente familiar positivo, permite la comprensión de los integrantes de la familia, el estímulo, la exigencia razonable. Las personas que se desarrollan en un clima positivo se sienten integrados y adaptados en la sociedad (Núñez, 2009). Serían los padres entonces quienes promueven la paz y la tolerancia en sus hijos, establecen leyes en la familia y roles dentro de la ella (Chong, 2015). De esta forma es muy importante la comunicación en el seno de la familia, donde se fortalecen los vínculos entre sus miembros, se debe fomentar la armonía, tolerancia, cooperación y un clima positivo entre sus integrantes (Castro, 2015), por medio de darse apoyo, confianza, respetar la intimidad, alentar autoestima, etc. Desde la perspectiva de Bronfenbrenner (1992; 2004) el microsistema familiar constituye un contexto crítico por sí mismo, ya que es aquí donde se aprenden las relaciones con las personas. Es cuando el individuo presta atención a las actividades de los demás y participa en ellas. El aprendizaje de las relaciones bidireccionales y sus reglas permite la formación de estructuras de orden superior en el complejo entramado de la sociedad y sus diversas instituciones.

Una de las instituciones donde las personas se insertan posteriormente a la familia es la escuela, lugar donde desarrollan habilidades, conocimientos, destrezas y actitudes que les permiten continuar con su formación social y académica. La escuela representa la institución formal donde el niño lleva a cabo el primer contacto en un contexto de relaciones sociales organizadas a través de autoridades y sistemas. En este contexto se lleva a cabo el proceso de enseñanza-aprendizaje, es el lugar donde se presentan interacciones sociales significativas de los alumnos con sus compañeros y con los maestros. La interacción entre las reglas de la institución y las relaciones entre las personas que allí interactúan crean el clima escolar.

En el caso de la educación media superior - Bachillerato, es una etapa importante en la vida de los jóvenes, ya que se considera una etapa de transición a la adultez. En esta etapa se delinean aspectos de la personalidad como el asertividad, la autoestima, aspectos clave para el desarrollo social de las personas. En este nivel educativo se espera se tomen decisiones, como la de continuar con sus estudios profesionales, conseguir un empleo - abandonar sus estudios. A la par, las instituciones educativas permiten el desarrollo 
de los estudiantes que se convertirán en los futuros profesionales, en un entorno globalizado y competitivo (García-Lastra, 2013).

El rendimiento académico ha sido entendido de diferentes formas. Por un lado, se piensa que es el resultado de la convivencia, de las actitudes y comportamientos de los alumnos con sus compañeros y maestros. Otros lo interpretan como una evaluación de exámenes y trabajos realizados para obtener promedios de determinadas asignaturas. Para algunos autores el rendimiento académico implica que los alumnos desarrollen cambios, acumulen conocimiento y aprendan habilidades para resolver problemas de acuerdo con los lineamientos establecidos por las diferentes instituciones (Rodríguez, 2011). Otras perspectivas señalan la importancia de medir el rendimiento académico, por medio de asignar un número que represente el nivel de desarrollo de los alumnos, en términos generales un promedio de las diferentes asignaturas al término de un periodo determinado (Morales, Morales y Holguín, 2016).

Para Solano (2015), el rendimiento académico tiene que ver con el nivel de conocimiento que los alumnos muestran tener en la materia que es evaluada. Se refiere al rendimiento que el alumno demuestra tener en las áreas, materias, asignaturas, en relación a los objetivos de aprendizaje y en comparación con los demás alumnos del grupo o aula. Para otros estudiosos en el rendimiento académico intervienen diferentes factores como el intelectual, la personalidad, la motivación, las aptitudes, los intereses, los hábitos de estudio, la autoestima o la relación profesor-alumno, entre otros elementos (Barreto, 2017).

En este trabajo se conceptualiza el rendimiento académico como el resultado en una calificación numérica, producto de los diferentes programas de estudio, el proceso de aprendizaje, la suma de los resultados de exámenes presentados, las actividades y destrezas desarrolladas en un ciclo escolar (Lamas, 2015).

En algunos estudios encaminados a conocer la relación entre la familia y el rendimiento académico encontramos los que señalan la importancia del nivel socioeconómico o educativo de la familia (Coleman 1966). Otros estudios destacan el grado y estilo de ayuda familiar a los hijos y la dinámica de las relaciones afectivas y de comunicación (Marchesi y Martín, 2002). Algunos han explorado las prácticas educativas desarrolladas por los padres haciendo énfasis en el interés por el proceso educativo de sus hijos y el apoyo familiar (Solís y Aguiar, 2017). Asimismo se ha señalado la importancia de ofrecer a los hijos una disciplina apropiada además de los recursos adecuados y necesarios en el ámbito escolar (Buote, 2001); una adecuada cohesión familiar (Caplan, Henderson, Henderson y Fleming, 2002) y un estilo parental basado en el apoyo y la solidaridad (Oliva, Parra y Arranz, 2008; Pelegrina, García y Casanova, 2002).

Varias investigaciones han informado que el nivel educativo de los padres incide en el rendimiento académico de los jóvenes. Cuando los padres presentan índices de analfabetismo es más probable que los resultados académicos de sus hijos no sean satisfactorios, ya que no estimulan el logro escolar en los alumnos. Por otro lado se ha observado que cuando el rango de estudios de los padres es de un nivel medio o superior, los padres suelen estimular el aprendizaje escolar de los hijos y por ende presentan mejores resultados en el ámbito académico. Asimismo se ha observado que quienes presentan un bagaje cultural y lingüístico distinto o considerado inferior son personas más vulnerables al fracaso escolar (Saconne, 2017). Otros estudios señalan la influencia de los estilos parentales, donde los hijos con padres que tenían un estilo parental negligente o permisivo presentaron las calificaciones más bajas, en contraste con aquellos adolescentes cuyos padres tenían un estilo autoritario tuvieron las calificaciones más altas.

Hallazgos distintos muestran que los adolescentes con padres con un alto nivel de afecto y control son los que presentan un mayor interés hacia la escuela y un mejor rendimiento académico. Por otro 
lado, señalan que los adolescentes que viven en hogares en los que predomina un estilo parental caracterizado por la falta de afecto y supervisión, manifiestan más problemas escolares, conductuales y de ajuste psicológico (Oliva, Parra, y Arranz, 2008). Por otra parte están los que han encontrado que es más importante la dinámica familiar que si los alumnos provienen de familias nucleares - monoparentales, argumentando que las relaciones que se desarrollan en la familia afectan los logros escolares en los jóvenes estudiantes (Sánchez, y Valdés, 2011). Sin embargo, esta incidencia positiva, no se presenta en todos los casos. Por ejemplo, en algunos trabajos en los que se realizó comparaciones en distintos grupos étnicos o culturales no se observó que los hijos de padres con un estilo parental caracterizado por el afecto y el control obtengan mejores resultados académicos (Chao, 2001; Garg, Levin, Urajnik, y Kauppi, 2005; Pittman y Chase-Lansdale, 2001).

Como se ha expuesto, el clima familiar caracterizado por un ambiente matizado por el afecto positivo, la comunicación, la seguridad, el respeto, la participación y la autonomía puede favorecer positivamente al rendimiento académico de los jóvenes. Un clima familiar con relaciones donde está ausente el dialogo, el afecto, la seguridad, donde se presente la rigidez de roles, puede significar una causa que lleve a altos índices de reprobación, fracaso escolar, deserción escolar y dificultades psicológicas en los alumnos, ya sean niños o adolescentes (Claro, 2013). Se entiende así que el clima familiar influirá de forma positiva o negativa en el proceso de aprendizaje en los alumnos.

Considerando todo lo expuesto anteriormente y que el índice de reprobación en educación media superior en México es de $37.4 \%$, el cual es muy superior al del nivel de secundaria $19.2 \%$ y al de educación primaria $5.2 \%$ (INEE, 2019) y la poca investigación entre el clima familiar y el rendimiento académico en nuestro contexto, Monterrey, México, juzgamos relevante indagar respecto a relación entre el clima familiar y el rendimiento académico, asimismo cuáles de las dimensiones del clima familiar se asocian a los distintos niveles de desempeño escolar de los jóvenes estudiantes de preparatoria. Partimos del supuesto, basado en los antecedentes expuestos párrafos arriba, que un clima familiar negativo se asociará a un bajo rendimiento académico. Consideramos que obtener conocimiento más profundo sobre las variables del clima familiar en el proceso del desarrollo escolar en estudiantes preparatoria permitirá disponer de información relevante para desarrollar programas de atención educativa, tomando en cuenta la comunicación y relación entre alumnos y sus padres. Esperando que intervenciones de este tipo permitan mejorar el desarrollo escolar de los jóvenes y por consiguiente el fracaso y la deserción escolar.

\section{MÉTODO}

\section{PARTICIPANTES}

Los participantes fueron elegidos de las preparatorias pertenecientes a la Universidad Autónoma de Nuevo León (México), esto por ser la institución responsable mayoritariamente de los estudios de nivel medio superior públicos en el estado. La selección fue por conveniencia en función del consentimiento de las autoridades escolares, así como de los padres y los estudiantes. Se tuvo acceso a 3 centros educativos y 290 estudiantes de $2^{\circ}$, $4^{\circ}$ y $6^{\circ}$ semestre de Bachillerato, con edades comprendidas entre 15 a 22 años, $(M=$ 16.36; DT $=0.06)$. El $49 \%$ eran mujeres $(n=$ $142)$ y el $51 \%$ hombres $(n=148)$.

\section{INSTRUMENTOS DE MEDIDA}

\section{Clima Familiar}

Para evaluar el clima psicosocial en la familia se revisó la Escala de Clima Social en la Familia (FES, Moss, Moss \& Trickett, 1984). La escala original cuenta con 90 ítems, los cuales se miden a través de una escala dicotómica de falso y verdadero. En nuestro estudio se empleó la versión reducida de la Escala de Clima Social en la 
Familia desarrollada Fernández-Ballesteros y Sierra (1989), la cual consta de 30 ítems los cuales miden las conductas de los padres, la interacción padres-hijos y la interacción entre hermanos.

Para esta investigación, se realizó una modificación en la forma de evaluar los reactivos, de la opción dicotómica original de la escala, a una escala tipo Likert con cinco opciones de respuesta (de $1=$ Nunca a 5 $=$ Siempre), esto con el objetivo de obtener posicionamientos más específicos de los estudiantes respecto a los enunciados. La subescala de interacción familiar positiva $(\alpha=.774)$ está compuesta por 8 ítems, evalúa la interacción positiva en la familia (p. ej., "En mi familia nos ayudamos y apoyamos realmente unos a otros"). La subescala de interacción familiar negativa $(\alpha=.634)$ consta de 7 ítems y evalúa la interacción negativa en la familia (p. ej., "En nuestra familia a veces discutimos"). Independencia familiar $\alpha=.546$ ) consta de 5 ítems y evalúa las acciones independientes de los miembros de la familia (p. ej., "En mi familia nos esforzamos mucho para mantener la independencia de cada uno"). Religiosidad ( $\alpha=.531$ ) consta de 4 ítems y evalúa la religiosidad en la familia ( $p$. ej., "Los miembros de mi familia asistimos con bastante regularidad a los cultos de la Iglesia, templo"). Socialización de la familia $(\alpha=$ .525), 3 ítems, evalúa como la familia socializa con los demás (p. ej., "Frecuentemente vienen amigos a comer a casa o a visitarnos"). La consistencia interna de la escala completa presenta un $\alpha$ de .718.

\section{Rendimiento académico}

Para esta variable se tomaron en cuenta el promedio general de los participantes, a través del Sistema de Información y Administración de Servicios Escolares (SIASE) de tres preparatorias públicas del Estado de Nuevo León, México. Dicho promedio se obtuvo gracias a la participación de las instituciones educativas previo consentimiento de las autoridades escolares, así como del consentimiento de los padres y estudiantes. Así la frecuencia y porcentaje de promedio general (SIASE) se categorizaron en calificaciones menores a 69, las cuales indican que los alumnos han reprobado el curso; calificaciones entre 70 y 79; las calificaciones entre 80 y 89 ; y calificaciones arriba de 90, que sería el intervalo de mejor rendimiento académico. Esta categorización obedece a que las calificaciones oficiales cuando es menor a 70 es una calificación no aprobatoria, cuando la calificación es mayor a 70 es una calificación aprobada, para la posterior estratificación es para señalar niveles cualitativos de rendimiento académico.

\section{PROCEDIMIENTO}

Se realizaron reuniones con las autoridades de las dependencias para exponer los objetivos del estudio, el procedimiento y el alcance de la investigación y obtener los permisos correspondientes. Se estableció la necesidad de contar con la aprobación expresa de los padres o tutores a través de un documento firmado por los mismos. Cumpliendo de esta forma con las exigencias deontológicas indicadas para investigación con menores de edad.

Se estableció una fecha para la aplicación de la escala. Dicha aplicación se llevó a cabo por los investigadores, en horario y las aulas habituales de los alumnos, sin presentarse incidentes. La aplicación duro alrededor de 30 minutos. En todos los casos, la participación fue voluntaria y anónima.

\section{ANÁLISIS DE DATOS}

Se realizaron análisis descriptivos para observar los promedios de las calificaciones, así como los rangos de edades y cantidad de alumnos por género. Se realizaron análisis de varianza (ANOVA) para estudiar la existencia de diferencias en cuanto a las variables de clima familiar y rendimiento académico. Se realizó la prueba t de las variables de clima familiar en función del género, para observar diferencias entre los tipos de relaciones familiares reportadas. Estos análisis se llevaron a cabo con el programa SPSS versión 25.0. 


\section{RESULTADOS}

Se realizaron una serie de análisis de medidas de tendencia central con las variables de estudio cuyos resultados se muestran a continuación.

En la Tabla 1 se muestra la frecuencia y porcentaje de promedio general (SIASE), donde se agruparon los promedios de los alumnos. Las calificaciones menores a 69 obtuvieron un porcentaje del $24.1 \%$, calificaciones entre 70 y 79 el $28.6 \%$, las calificaciones entre 80 y 89 el 40.3\%, calificaciones arriba de 90 el $6.9 \%$.
Una vez observado los resultados descriptivos de las variables se realizó un análisis de correlación entre las calificacionesy las subescalas de clima familiar (Tabla 2). La variable Calificaciones de SIASE tiene una correlación negativa y significativa con la Interacción familiar y con la Independencia familiar; por otro lado, encontramos una correlación significativa positiva, entre Interacción familiar positiva y Religiosidad, Socialización de la familia, e Independencia familiar. Se observa también una correlación positiva significativa entre Interacción familiar negativa con Independencia familiar y con Socialización de la familia.

Tabla 1

Frecuencia y porcentaje de los promedios de calificaciones

\begin{tabular}{|c|c|c|}
\hline $\begin{array}{c}\text { Promedio general de } \\
\text { calificaciones }\end{array}$ & Frecuencia & Porcentaje \\
\hline Menor de 69 & 70 & 24.1 \\
\hline De 70 a 79 & 83 & 28.6 \\
\hline De 80 a 89 & 117 & 40.3 \\
\hline De 90 a 100 & 20 & 6.9 \\
\hline Total & 290 & 100 \\
\hline
\end{tabular}

Tabla 2

Correlación de Pearson de las calificaciones de SIASE con las variables de Clima Familiar (N=290)

\begin{tabular}{|c|c|c|c|c|c|c|}
\hline & & 1 & 2 & 3 & 4 & 5 \\
\hline \multirow[t]{2}{*}{ 1. Calificación de SIASE } & $r$ & & & & & \\
\hline & Sig. & & & & & \\
\hline \multirow[t]{2}{*}{ 2. Interacción Familiar Positiva } & $r$ & .095 & & & & \\
\hline & Sig. & .107 & & & & \\
\hline \multirow[t]{2}{*}{ 3. Interacción Familiar Negativa } & r & $-.224^{\star \star}$ & -.059 & & & \\
\hline & Sig. & $<.001$ & .307 & & & \\
\hline \multirow[t]{2}{*}{ 4. Independencia Familiar } & $r$ & $-.141 *$ & $.127 \star$ & $.212^{\star \star}$ & & \\
\hline & Sig. & .016 & .027 & $<.001$ & & \\
\hline \multirow[t]{2}{*}{ 5. Religiosidad } & $r$ & -.062 & $.378^{\star *}$ & -.029 & $.159 * \star$ & \\
\hline & Sig. & .291 & $<.001$ & .615 & .006 & \\
\hline \multirow[t]{2}{*}{ 6. Socialización de la Familia } & r & -.099 & $.171^{* \star}$ & $.224^{\star *}$ & .060 & $.235^{\star *}$ \\
\hline & Sig. & .091 & .003 & $<.001$ & .296 & $<.001$ \\
\hline
\end{tabular}


Posteriormente se realizó el ANOVA con las variables de Clima Familiar, en contraste con los promedios de calificación de los alumnos. Las tablas 3 y 4 muestran los resultados. En la Tabla 3, los resultados descriptivos de ANOVA nos muestran una tendencia respecto a que aquellos que tenían mayores calificaciones por lo general mostraron medias más altas en cada reactivo contrastado. Por contraparte, aquellos con calificaciones más bajas tendieron a obtener medias más bajas, aunque no todos llegaron a mostrar diferencias significativas como lo veremos enseguida.
En la Tabla 4 se observa en qué reactivos se encontraron diferencias estadísticamente significativas entre las variables contrastadas y los grupos (los promedios de calificaciones): en el reactivo "los miembros de la familia a menudo guardan sentimientos para símismos", donde quienes más guardan los sentimientos son los alumnos con menores calificaciones y quienes menos guardan los sentimientos son los de mayores calificaciones; "en nuestra familia a veces discutimos", donde quienes más reportan discutir en familia son los alumnos con menores calificaciones y quienes menos reportan discutir en familia son los de mayores calificaciones. Esta tendencia de

Tabla 3

Descriptivos de ANOVA de un factor de las calificaciones de SIASE con las variables Clima Familiar

\begin{tabular}{|c|c|c|c|c|c|c|c|c|c|}
\hline & & & & & & \multicolumn{2}{|c|}{ IC 95\% } & \multirow[b]{2}{*}{ Mín. } & \multirow[b]{2}{*}{ Max. } \\
\hline & & $\mathrm{n}$ & $M$ & DT & ET & LI & LS & & \\
\hline \multirow{5}{*}{$\begin{array}{l}\text { En mi familia } \\
\text { nos ayudamos } \\
\text { y apoyamos } \\
\text { realmente unos a } \\
\text { otros. }\end{array}$} & Menor de 69 & 70 & 4.03 & 1.08 & .13 & 3.77 & 4.29 & 1 & 5 \\
\hline & De 70 a 79 & 83 & 4.13 & 0.84 & .09 & 3.95 & 4.32 & 2 & 5 \\
\hline & De 80 a 89 & 117 & 4.31 & 0.84 & .08 & 4.15 & 4.46 & 2 & 5 \\
\hline & De 90 a 100 & 20 & 4.35 & 0.81 & .18 & 3.97 & 4.73 & 2 & 5 \\
\hline & Total & 290 & 4.19 & 0.90 & .05 & 4.09 & 4.30 & 1 & 5 \\
\hline \multirow{5}{*}{$\begin{array}{l}\text { Las actividades } \\
\text { de nuestra familia } \\
\text { se planifican } \\
\text { cuidadosamente. }\end{array}$} & Menor de 69 & 70 & 3.07 & 1.17 & .14 & 2.79 & 3.35 & 1 & 5 \\
\hline & De 70 a 79 & 83 & 3.14 & 1.18 & .13 & 2.89 & 3.40 & 1 & 5 \\
\hline & De 80 a 89 & 117 & 3.03 & 1.12 & .10 & 2.82 & 3.23 & 1 & 5 \\
\hline & De 90 a 100 & 20 & 3.30 & 1.08 & .24 & 2.79 & 3.81 & 2 & 5 \\
\hline & Total & 290 & 3.09 & 1.14 & .07 & 2.96 & 3.22 & 1 & 5 \\
\hline \multirow{5}{*}{$\begin{array}{l}\text { En mi familia } \\
\text { tenemos } \\
\text { situaciones en } \\
\text { las que debemos } \\
\text { reunirnos de forma } \\
\text { obligatoria todos. }\end{array}$} & Menor de 69 & 70 & 3.07 & 1.33 & .16 & 2.75 & 3.39 & 1 & 5 \\
\hline & De 70 a 79 & 83 & 3.00 & 1.16 & .13 & 2.75 & 3.25 & 1 & 5 \\
\hline & De 80 a 89 & 117 & 3.12 & 1.09 & .10 & 2.92 & 3.32 & 1 & 5 \\
\hline & De 90 a 100 & 20 & 3.15 & 1.14 & .25 & 2.62 & 3.68 & 1 & 5 \\
\hline & Total & 290 & 3.08 & 1.17 & .07 & 2.94 & 3.21 & 1 & 5 \\
\hline
\end{tabular}


Tabla 3 (Continuación)

Descriptivos de ANOVA de un factor de las calificaciones de SIASE con las variables Clima Familiar

\begin{tabular}{|c|c|c|c|c|c|c|c|c|c|}
\hline & & \multirow[b]{2}{*}{$\mathrm{n}$} & \multirow[b]{2}{*}{$M$} & \multirow[b]{2}{*}{ DT } & \multirow[b]{2}{*}{ ET } & \multicolumn{2}{|c|}{ IC 95\% } & \multirow[b]{2}{*}{ Mín. } & \multirow[b]{2}{*}{ Max } \\
\hline & & & & & & LI & LS & & \\
\hline \multirow{5}{*}{$\begin{array}{l}\text { En casa hablamos } \\
\text { abiertamente de lo } \\
\text { que nos parece o } \\
\text { queremos. }\end{array}$} & $\begin{array}{c}\text { Menor de } \\
69\end{array}$ & 70 & 3.74 & 1.26 & .15 & 3.44 & 4.04 & 1 & 5 \\
\hline & $\begin{array}{c}\text { De } 70 \text { a } \\
79\end{array}$ & 83 & 3.92 & 1.07 & .12 & 3.68 & 4.15 & 1 & 5 \\
\hline & $\begin{array}{c}\text { De } 80 \text { a } \\
89\end{array}$ & 117 & 3.85 & 1.00 & .09 & 3.67 & 4.04 & 1 & 5 \\
\hline & $\begin{array}{c}\text { De } 90 \text { a } \\
100\end{array}$ & 20 & 3.90 & 0.91 & .20 & 3.47 & 4.33 & 2 & 5 \\
\hline & Total & 290 & 3.85 & 1.08 & .06 & 3.72 & 3.97 & 1 & 5 \\
\hline \multirow{5}{*}{$\begin{array}{l}\text { Para mi familia es } \\
\text { muy importante } \\
\text { triunfar en la vida. }\end{array}$} & $\begin{array}{c}\text { Menor de } \\
69\end{array}$ & 70 & 4.27 & 0.90 & .11 & 4.06 & 4.49 & 1 & 5 \\
\hline & $\begin{array}{c}\text { De } 70 \text { a } \\
79\end{array}$ & 83 & 4.33 & 0.78 & .09 & 4.15 & 4.50 & 2 & 5 \\
\hline & $\begin{array}{c}\text { De } 80 \text { a } \\
89\end{array}$ & 117 & 4.40 & 0.75 & .07 & 4.26 & 4.54 & 2 & 5 \\
\hline & $\begin{array}{c}\text { De } 90 \text { a } \\
100\end{array}$ & 20 & 4.25 & 1.02 & .23 & 3.77 & 4.73 & 2 & 5 \\
\hline & Total & 290 & 4.34 & 0.82 & .05 & 4.24 & 4.43 & 1 & 5 \\
\hline \multirow{5}{*}{$\begin{array}{l}\text { En mi casa somos } \\
\text { muy ordenados y } \\
\text { limpios. }\end{array}$} & $\begin{array}{c}\text { Menor de } \\
69\end{array}$ & 70 & 3.74 & 1.13 & .13 & 3.47 & 4.01 & 1 & 5 \\
\hline & $\begin{array}{c}\text { De } 70 \text { a } \\
79\end{array}$ & 83 & 3.55 & 1.07 & .12 & 3.32 & 3.79 & 1 & 5 \\
\hline & $\begin{array}{c}\text { De } 80 \text { a } \\
89\end{array}$ & 117 & 3.76 & 0.98 & .09 & 3.58 & 3.94 & 1 & 5 \\
\hline & $\begin{array}{c}\text { De } 90 \text { a } \\
100\end{array}$ & 20 & 3.95 & 1.00 & .22 & 3.48 & 4.42 & 2 & 5 \\
\hline & Total & 290 & 3.71 & 1.04 & .06 & 3.59 & 3.83 & 1 & 5 \\
\hline \multirow{5}{*}{$\begin{array}{c}\text { Todos nos } \\
\text { esforzamos mucho } \\
\text { en lo que hacemos } \\
\text { en casa. }\end{array}$} & $\begin{array}{c}\text { Menor de } \\
69\end{array}$ & 70 & 3.81 & 1.03 & .12 & 3.57 & 4.06 & 1 & 5 \\
\hline & $\begin{array}{c}\text { De } 70 \text { a } \\
79\end{array}$ & 83 & 3.73 & 0.99 & .11 & 3.52 & 3.95 & 1 & 5 \\
\hline & $\begin{array}{c}\text { De } 80 \text { a } \\
89\end{array}$ & 117 & 3.95 & 0.87 & .08 & 3.79 & 4.11 & 2 & 5 \\
\hline & $\begin{array}{c}\text { De } 90 \text { a } \\
100\end{array}$ & 20 & 4.10 & 0.64 & .14 & 3.80 & 4.40 & 2 & 5 \\
\hline & Total & 290 & 3.87 & 0.93 & .05 & 3.76 & 3.97 & 1 & 5 \\
\hline \multirow{5}{*}{$\begin{array}{l}\text { En mi familia es } \\
\text { muy importante } \\
\text { aprender algo } \\
\text { nuevo o diferente }\end{array}$} & $\begin{array}{c}\text { Menor de } \\
69\end{array}$ & 70 & 3.83 & 0.98 & .12 & 3.60 & 4.06 & 1 & 5 \\
\hline & $\begin{array}{c}\text { De } 70 \text { a } \\
79\end{array}$ & 83 & 3.86 & 0.95 & .10 & 3.65 & 4.06 & 1 & 5 \\
\hline & $\begin{array}{c}\text { De } 80 \text { a } \\
89\end{array}$ & 117 & 4.00 & 0.92 & .08 & 3.83 & 4.17 & 1 & 5 \\
\hline & $\begin{array}{c}\text { De } 90 \text { a } \\
100\end{array}$ & 20 & 4.30 & 0.73 & .16 & 3.96 & 4.64 & 3 & 5 \\
\hline & Total & 290 & 3.94 & 0.94 & .05 & 3.83 & 4.05 & 1 & 5 \\
\hline
\end{tabular}


Tabla 3 (Continuación)

Descriptivos de ANOVA de un factor de las calificaciones de SIASE con las variables Clima Familiar

\begin{tabular}{|c|c|c|c|c|c|c|c|c|c|}
\hline & & \multirow[b]{2}{*}{$\mathrm{n}$} & \multirow[b]{2}{*}{$M$} & \multirow[b]{2}{*}{ DT } & \multirow[b]{2}{*}{ ET } & \multicolumn{2}{|c|}{ IC 95\% } & \multirow[b]{2}{*}{ Mín. } & \multirow[b]{2}{*}{ Max. } \\
\hline & & & & & & LI & LS & & \\
\hline \multirow{5}{*}{$\begin{array}{l}\text { Los miembros de } \\
\text { la familia guardan, } \\
\text { a menudo, } \\
\text { sentimientos para si } \\
\text { mismos. }\end{array}$} & $\begin{array}{l}\text { Menor de } \\
69\end{array}$ & 70 & 3.53 & 1.25 & .15 & 3.23 & 3.83 & 1 & 5 \\
\hline & $\begin{array}{c}\text { De } 70 \text { a } \\
79\end{array}$ & 83 & 3.29 & 0.96 & .11 & 3.08 & 3.50 & 1 & 5 \\
\hline & $\begin{array}{c}\text { De } 80 \text { a } \\
89\end{array}$ & 117 & 3.15 & 0.98 & .09 & 2.97 & 3.33 & 1 & 5 \\
\hline & $\begin{array}{l}\text { De } 90 \text { a } \\
\quad 100\end{array}$ & 20 & 2.95 & 0.83 & .18 & 2.56 & 3.34 & 2 & 4 \\
\hline & Total & 290 & 3.27 & 1.04 & .06 & 3.15 & 3.39 & 1 & 5 \\
\hline \multirow{5}{*}{$\begin{array}{l}\text { En nuestra familia a } \\
\text { veces discutimos. }\end{array}$} & $\begin{array}{l}\text { Menor de } \\
69\end{array}$ & 70 & 3.74 & 1.13 & .13 & 3.47 & 4.01 & 1 & 5 \\
\hline & $\begin{array}{c}\text { De } 70 \text { a } \\
79\end{array}$ & 83 & 3.42 & 1.17 & .13 & 3.17 & 3.68 & 1 & 5 \\
\hline & $\begin{array}{c}\mathrm{De} 80 \mathrm{a} \\
89\end{array}$ & 117 & 3.34 & 1.06 & .10 & 3.15 & 3.54 & 1 & 5 \\
\hline & $\begin{array}{l}\text { De } 90 \text { a } \\
100\end{array}$ & 20 & 3.05 & 1.00 & .22 & 2.58 & 3.52 & 1 & 5 \\
\hline & Total & 290 & 3.44 & 1.12 & .07 & 3.31 & 3.57 & 1 & 5 \\
\hline \multirow{5}{*}{$\begin{array}{l}\text { En general ningún } \\
\text { miembro de la } \\
\text { familia decide por } \\
\text { su cuenta. }\end{array}$} & $\begin{array}{l}\text { Menor de } \\
69\end{array}$ & 70 & 3.30 & 1.20 & .14 & 3.01 & 3.59 & 1 & 5 \\
\hline & $\begin{array}{l}\text { De } 70 \text { a } \\
79\end{array}$ & 83 & 2.99 & 0.96 & .10 & 2.78 & 3.20 & 1 & 5 \\
\hline & $\begin{array}{c}\text { De } 80 \text { a } \\
89\end{array}$ & 117 & 2.88 & 0.98 & .09 & 2.70 & 3.06 & 1 & 5 \\
\hline & $\begin{array}{l}\text { De } 90 \text { a } \\
100\end{array}$ & 20 & 2.65 & 1.09 & .24 & 2.14 & 3.16 & 1 & 4 \\
\hline & Total & 290 & 3.00 & 1.05 & .06 & 2.88 & 3.12 & 1 & 5 \\
\hline \multirow{5}{*}{$\begin{array}{c}\text { En casa solo } \\
\text { estamos pasando } \\
\text { el rato. }\end{array}$} & $\begin{array}{l}\text { Menor de } \\
69\end{array}$ & 70 & 3.16 & 1.14 & .14 & 2.89 & 3.43 & 1 & 5 \\
\hline & $\begin{array}{c}\text { De } 70 \text { a } \\
79\end{array}$ & 83 & 3.12 & 1.11 & .12 & 2.88 & 3.36 & 1 & 5 \\
\hline & $\begin{array}{c}\text { De } 80 \text { a } \\
89\end{array}$ & 117 & 2.99 & 1.06 & .10 & 2.80 & 3.19 & 1 & 5 \\
\hline & $\begin{array}{l}\text { De } 90 \text { a } \\
100\end{array}$ & 20 & 2.65 & 0.99 & .22 & 2.19 & 3.11 & 1 & 5 \\
\hline & Total & 290 & 3.04 & 1.09 & .06 & 2.92 & 3.17 & 1 & 5 \\
\hline \multirow{5}{*}{$\begin{array}{l}\text { Cuando me quejo } \\
\text { de algo que me } \\
\text { molesta se enojan } \\
\text { en mi familia. }\end{array}$} & $\begin{array}{l}\text { Menor de } \\
69\end{array}$ & 70 & 3.20 & 1.38 & .16 & 2.87 & 3.53 & 1 & 5 \\
\hline & $\begin{array}{c}\text { De } 70 \text { a } \\
79\end{array}$ & 83 & 2.98 & 1.17 & .13 & 2.73 & 3.23 & 1 & 5 \\
\hline & $\begin{array}{c}\text { De } 80 \text { a } \\
89\end{array}$ & 117 & 2.96 & 2.13 & .20 & 2.57 & 3.35 & 1 & 5 \\
\hline & $\begin{array}{l}\text { De } 90 \text { a } \\
100\end{array}$ & 20 & 2.90 & 1.17 & .26 & 2.35 & 3.45 & 1 & 5 \\
\hline & Total & 290 & 3.02 & 1.66 & 10 & 2.83 & 3.21 & 1 & 5 \\
\hline
\end{tabular}


Tabla 3 (Continuación)

Descriptivos de ANOVA de un factor de las calificaciones de SIASE con las variables Clima Familiar

\begin{tabular}{|c|c|c|c|c|c|c|c|c|c|}
\hline & & \multirow[b]{2}{*}{$\mathrm{n}$} & \multirow[b]{2}{*}{ M } & \multirow[b]{2}{*}{ DT } & \multirow[b]{2}{*}{ ET } & \multicolumn{2}{|c|}{ IC 95\% } & \multirow[b]{2}{*}{ Mín. } & \multirow[b]{2}{*}{ Max } \\
\hline & & & & & & LI & LS & & \\
\hline \multirow{5}{*}{$\begin{array}{l}\text { En mi casa a veces } \\
\text { los miembros } \\
\text { de la familia } \\
\text { discutimos y nos } \\
\text { enojamos tanto } \\
\text { que golpeamos o } \\
\text { rompemos cosas. }\end{array}$} & Menor de 69 & 70 & 2.39 & 1.33 & .16 & 2.07 & 2.70 & 1 & 5 \\
\hline & De 70 a 79 & 83 & 2.13 & 1.26 & .14 & 1.86 & 2.41 & 1 & 5 \\
\hline & De 80 a 89 & 117 & 1.74 & 1.06 & .10 & 1.54 & 1.93 & 1 & 5 \\
\hline & De 90 a 100 & 20 & 2.00 & 1.12 & .25 & 1.47 & 2.53 & 1 & 5 \\
\hline & Total & 290 & 2.02 & 1.22 & .07 & 1.88 & 2.16 & 1 & 5 \\
\hline \multirow{5}{*}{$\begin{array}{l}\text { En mi casa una } \\
\text { sola persona toma } \\
\text { la mayoría de las } \\
\text { decisiones. }\end{array}$} & Menor de 69 & 70 & 3.00 & 1.27 & .15 & 2.70 & 3.30 & 1 & 5 \\
\hline & De 70 a 79 & 83 & 2.94 & 1.28 & .14 & 2.66 & 3.22 & 1 & 5 \\
\hline & De 80 a 89 & 117 & 2.52 & 1.16 & .11 & 2.31 & 2.73 & 1 & 5 \\
\hline & De 90 a 100 & 20 & 2.45 & 1.23 & .28 & 1.87 & 3.03 & 1 & 5 \\
\hline & Total & 290 & 2.75 & 1.24 & .07 & 2.61 & 2.90 & 1 & 5 \\
\hline
\end{tabular}

Tabla 4

ANOVA de un factor de Calificaciones de SIASE en contraste con la variable clima familiar

\begin{tabular}{|c|c|c|c|c|c|c|}
\hline & & $\begin{array}{l}\text { Suma de } \\
\text { cuadrados }\end{array}$ & gl & $\begin{array}{c}\text { Media } \\
\text { cuadrática }\end{array}$ & $F$ & Sig. \\
\hline \multirow{3}{*}{$\begin{array}{l}\text { En mi familia nos ayudamos y } \\
\text { apoyamos realmente unos a otros. }\end{array}$} & Inter-grupos & 4.228 & 3 & 1.409 & 1.745 & .158 \\
\hline & Intra-grupos & 230.958 & 286 & .808 & & \\
\hline & Total & 235.186 & 289 & & & \\
\hline \multirow{3}{*}{$\begin{array}{l}\text { Las actividades de nuestra familia } \\
\text { se planifican cuidadosamente. }\end{array}$} & Inter-grupos & 1.638 & 3 & .546 & .415 & .742 \\
\hline & Intra-grupos & 376.031 & 286 & 1.315 & & \\
\hline & Total & 377.669 & 289 & & & \\
\hline \multirow{3}{*}{$\begin{array}{l}\text { En mi familia tenemos situaciones } \\
\text { en las que debemos reunirnos de } \\
\text { forma obligatoria todos. }\end{array}$} & Inter-grupos & .813 & 3 & .271 & .196 & .899 \\
\hline & Intra-grupos & 395.518 & 286 & 1.383 & & \\
\hline & Total & 396.331 & 289 & & & \\
\hline \multirow{3}{*}{$\begin{array}{c}\text { En casa hablamos abiertamente } \\
\text { de lo que nos parece o } \\
\text { queremos. }\end{array}$} & Inter-grupos & 1.213 & 3 & .404 & .344 & .793 \\
\hline & Intra-grupos & 336.111 & 286 & 1.175 & & \\
\hline & Total & 337.324 & 289 & & & \\
\hline \multirow{3}{*}{$\begin{array}{l}\text { Para mi familia es muy importante } \\
\text { triunfar en la vida. }\end{array}$} & Inter-grupos & .953 & 3 & .318 & .474 & .701 \\
\hline & Intra-grupos & 191.929 & 286 & .671 & & \\
\hline & Total & 192.883 & 289 & & & \\
\hline \multirow{3}{*}{$\begin{array}{l}\text { En mi casa somos muy ordenados } \\
\qquad y \text { limpios. }\end{array}$} & Inter-grupos & 3.542 & 3 & 1.181 & 1.082 & .357 \\
\hline & Intra-grupos & 312.127 & 286 & 1.091 & & \\
\hline & Total & 315.669 & 289 & & & \\
\hline \multirow{3}{*}{$\begin{array}{l}\text { Todos nos esforzamos mucho en } \\
\text { lo que hacemos en casa. }\end{array}$} & Inter-grupos & 3.508 & 3 & 1.169 & 1.347 & .259 \\
\hline & Intra-grupos & 248.247 & 286 & .868 & & \\
\hline & Total & 251.755 & 289 & & & \\
\hline
\end{tabular}


Tabla 4 (Continuación)

ANOVA de un factor de Calificaciones de SIASE en contraste con la variable clima familiar

\begin{tabular}{|c|c|c|c|c|c|c|}
\hline & & $\begin{array}{l}\text { Suma de } \\
\text { cuadrados }\end{array}$ & gl & $\begin{array}{c}\text { Media } \\
\text { cuadrática }\end{array}$ & $\mathrm{F}$ & Sig. \\
\hline \multirow{3}{*}{$\begin{array}{l}\text { En mi familia es muy } \\
\text { importante aprender } \\
\text { algo nuevo o diferente }\end{array}$} & Inter-grupos & 4.475 & 3 & 1.492 & 1.717 & .164 \\
\hline & Intra-grupos & 248.408 & 286 & .869 & & \\
\hline & Total & 252.883 & 289 & & & \\
\hline \multirow{3}{*}{$\begin{array}{l}\text { Los miembros de la } \\
\text { familia guardan, a } \\
\text { menudo, sentimientos } \\
\text { para sí mismos. }\end{array}$} & Inter-grupos & 8.337 & 3 & 2.779 & 2.592 & .053 \\
\hline & Intra-grupos & 306.684 & 286 & 1.072 & & \\
\hline & Total & 315.021 & 289 & & & \\
\hline \multirow{3}{*}{$\begin{array}{l}\text { En nuestra familia a } \\
\text { veces discutimos. }\end{array}$} & Inter-grupos & 10.616 & 3 & 3.539 & 2.901 & .035 \\
\hline & Intra-grupos & 348.887 & 286 & 1.220 & & \\
\hline & Total & 359.503 & 289 & & & \\
\hline \multirow{3}{*}{$\begin{array}{l}\text { En general ningún } \\
\text { miembro de la familia } \\
\text { decide por su cuenta. }\end{array}$} & Inter-grupos & 10.434 & 3 & 3.478 & 3.224 & .023 \\
\hline & Intra-grupos & 308.563 & 286 & 1.079 & & \\
\hline & Total & 318.997 & 289 & & & \\
\hline \multirow{3}{*}{$\begin{array}{c}\text { En casa solo estamos } \\
\text { pasando el rato. }\end{array}$} & Inter-grupos & 4.809 & 3 & 1.603 & 1.350 & .258 \\
\hline & Intra-grupos & 339.608 & 286 & 1.187 & & \\
\hline & Total & 344.417 & 289 & & & \\
\hline \multirow{3}{*}{$\begin{array}{l}\text { Cuando me quejo de } \\
\text { algo que me molesta } \\
\text { se enojan en mi familia. }\end{array}$} & Inter-grupos & 3.176 & 3 & 1.059 & .382 & .766 \\
\hline & Intra-grupos & 791.738 & 286 & 2.768 & & \\
\hline & Total & 794.914 & 289 & & & \\
\hline \multirow{3}{*}{$\begin{array}{l}\text { En mi casa a veces } \\
\text { los miembros de la } \\
\text { familia discutimos y } \\
\text { nos enojamos tanto } \\
\text { que golpeamos o } \\
\text { rompemos cosas. }\end{array}$} & Inter-grupos & 19.917 & 3 & 6.639 & 4.666 & .003 \\
\hline & Intra-grupos & 406.914 & 286 & 1.423 & & \\
\hline & Total & 426.831 & 289 & & & \\
\hline \multirow{3}{*}{$\begin{array}{l}\text { En mi casa una } \\
\text { sola persona toma } \\
\text { la mayoría de las } \\
\text { decisiones. }\end{array}$} & Inter-grupos & 15.279 & 3 & 5.093 & 3.381 & .019 \\
\hline & Intra-grupos & 430.845 & 286 & 1.506 & & \\
\hline & Total & 446.124 & 289 & & & \\
\hline
\end{tabular}

medias más altas y bajas entre los grupos de menores y mayores calificaciones también se encontró en los siguientes reactivos, donde se obtuvieron diferencias significativas: "en general ningún miembro de la familia decide por su cuenta"; "en mi casa a veces los miembros de la familia discutimos y nos enojamos tanto que golpeamos o rompemos cosas" y, por último, "en mi casa una sola persona toma la mayoría de las decisiones".
Con la finalidad de observar diferencias de medias entre las variables de clima familiar en función del sexo de los participantes, se realizó la Prueba $T$ con estas variables. En la tabla 5 se muestra los resultados.

La Tabla 6 muestra diferencias significativas entre los grupos de hombres y mujeres solo en algunos reactivos. Los varones presentan medias más altas en el reactivo "Las actividades de nuestra familia se planifican 
Tabla 5

Diferencias de medias en las variables de Clima Familiar en función del sexo de los participantes

\begin{tabular}{|c|c|c|c|c|c|}
\hline & Sexo & $\mathrm{n}$ & $\mathrm{M}$ & DT & ET \\
\hline \multirow{2}{*}{$\begin{array}{l}\text { En mi familia nos ayudamos y apoyamos } \\
\text { realmente unos a otros. }\end{array}$} & Masculino & 148 & 4.23 & 0.88 & .07 \\
\hline & Femenino & 142 & 4.12 & 0.97 & .08 \\
\hline \multirow{2}{*}{$\begin{array}{l}\text { Las actividades de nuestra familia se } \\
\text { planifican cuidadosamente. }\end{array}$} & Masculino & 148 & 3.22 & 1.06 & .09 \\
\hline & Femenino & 142 & 2.95 & 1.20 & .10 \\
\hline \multirow{2}{*}{$\begin{array}{l}\text { En mi familia tenemos situaciones en } \\
\text { las que debemos reunirnos de forma } \\
\text { obligatoria todos. }\end{array}$} & Masculino & 148 & 3.18 & 1.13 & .09 \\
\hline & Femenino & 142 & 2.95 & 1.18 & .10 \\
\hline \multirow{2}{*}{$\begin{array}{c}\text { En casa hablamos abiertamente de lo } \\
\text { que nos parece o queremos. }\end{array}$} & Masculino & 148 & 3.83 & 1.07 & .09 \\
\hline & Femenino & 142 & 3.85 & 1.09 & .09 \\
\hline \multirow{2}{*}{$\begin{array}{l}\text { Para mi familia es muy importante triunfar } \\
\text { en la vida. }\end{array}$} & Masculino & 148 & 4.40 & 0.81 & .07 \\
\hline & Femenino & 142 & 4.27 & 0.83 & .07 \\
\hline \multirow{2}{*}{$\begin{array}{l}\text { En mi casa somos muy ordenados y } \\
\text { limpios. }\end{array}$} & Masculino & 148 & 3.84 & 0.98 & .08 \\
\hline & Femenino & 142 & 3.54 & 1.10 & .09 \\
\hline \multirow{2}{*}{$\begin{array}{l}\text { Todos nos esforzamos mucho en lo que } \\
\text { hacemos en casa. }\end{array}$} & Masculino & 148 & 3.90 & 0.89 & .07 \\
\hline & Femenino & 142 & 3.80 & 0.99 & .08 \\
\hline \multirow{2}{*}{$\begin{array}{l}\text { En mi familia es muy importante aprender } \\
\text { algo nuevo o diferente }\end{array}$} & Masculino & 148 & 4.05 & 0.90 & .07 \\
\hline & Femenino & 142 & 3.86 & 0.98 & .08 \\
\hline \multirow{2}{*}{$\begin{array}{l}\text { Los miembros de la familia guardan, a } \\
\text { menudo, sentimientos para si mismos. }\end{array}$} & Masculino & 148 & 3.23 & 0.99 & .08 \\
\hline & Femenino & 142 & 3.30 & 1.09 & .09 \\
\hline \multirow{2}{*}{ En nuestra familia a veces discutimos. } & Masculino & 148 & 3.31 & 1.12 & .09 \\
\hline & Femenino & 142 & 3.60 & 1.08 & .09 \\
\hline \multirow{2}{*}{$\begin{array}{l}\text { En general ningún miembro de la familia } \\
\text { decide por su cuenta. }\end{array}$} & Masculino & 148 & 2.99 & 1.06 & .09 \\
\hline & Femenino & 142 & 2.99 & 1.05 & .09 \\
\hline \multirow{2}{*}{ En casa solo estamos pasando el rato. } & Masculino & 148 & 3.02 & 1.06 & .09 \\
\hline & Femenino & 142 & 3.05 & 1.14 & .09 \\
\hline \multirow{2}{*}{$\begin{array}{l}\text { Cuando me quejo de algo que me } \\
\text { molesta se enojan en mi familia. }\end{array}$} & Masculino & 148 & 3.11 & 1.98 & .16 \\
\hline & Femenino & 142 & 2.92 & 1.18 & .10 \\
\hline \multirow{2}{*}{$\begin{array}{l}\text { En mi casa a veces los miembros de la } \\
\text { familia discutimos y nos enojamos tanto } \\
\text { que golpeamos o rompemos cosas. }\end{array}$} & Masculino & 148 & 1.90 & 1.13 & .09 \\
\hline & Femenino & 142 & 2.12 & 1.27 & .10 \\
\hline \multirow{2}{*}{$\begin{array}{l}\text { En mi casa una sola persona toma la } \\
\text { mayoría de las decisiones. }\end{array}$} & Masculino & 148 & 2.72 & 1.23 & .10 \\
\hline & Femenino & 142 & 2.74 & 1.23 & .10 \\
\hline
\end{tabular}


Tabla 6

Prueba $T$ de muestras independientes

\begin{tabular}{|c|c|c|c|c|c|c|c|c|c|c|}
\hline & \multirow{3}{*}{$\begin{array}{l}\text { Se han } \\
\text { asumido } \\
\text { varianzas } \\
\text { iguales }\end{array}$} & \multicolumn{2}{|c|}{$\begin{array}{l}\text { Prueba de } \\
\text { Levene }\end{array}$} & \multirow[b]{3}{*}{$t$} & \multirow[b]{3}{*}{ gl } & \multirow[b]{3}{*}{ Sig. } & \multirow{3}{*}{$\begin{array}{l}\text { Prueba } \\
\qquad \mathrm{T} \\
\\
\text { Dif. de } \\
\text { Medias }\end{array}$} & \multirow[b]{3}{*}{ ET } & \multirow{2}{*}{\multicolumn{2}{|c|}{ IC 95\% }} \\
\hline & & \multirow[b]{2}{*}{$\mathrm{F}$} & \multirow[b]{2}{*}{ Sig. } & & & & & & & \\
\hline & & & & & & & & & LI & LS \\
\hline \multirow{2}{*}{$\begin{array}{c}\text { En mi familia nos ayudamos } \\
\text { y apoyamos realmente } \\
\text { unos a otros. }\end{array}$} & Sí & 0.567 & .45 & 1.063 & 301 & .289 & 0.11 & .11 & -.10 & .32 \\
\hline & No & & & 1.061 & 295.550 & .289 & 0.11 & .11 & -.10 & .32 \\
\hline \multirow{2}{*}{$\begin{array}{l}\text { Las actividades de nuestra } \\
\text { familia se planifican } \\
\text { cuidadosamente. }\end{array}$} & Sí & 0.713 & .40 & 2.060 & 301 & .040 & 0.27 & .13 & .01 & .52 \\
\hline & No & & & 2.056 & 294.064 & .041 & 0.27 & .13 & .01 & .52 \\
\hline \multirow{2}{*}{$\begin{array}{l}\text { En mi familia tenemos } \\
\text { situaciones en las que } \\
\text { debemos reunirnos de } \\
\text { forma obligatoria todos. }\end{array}$} & Sí & 0.008 & .93 & 1.724 & 301 & .086 & 0.23 & .13 & -.03 & .49 \\
\hline & No & & & 1.723 & 299.573 & .086 & 0.23 & .13 & -.03 & .49 \\
\hline \multirow{2}{*}{$\begin{array}{c}\text { En casa hablamos } \\
\text { abiertamente de lo que nos } \\
\text { parece o queremos. }\end{array}$} & Sí & 0.038 & .85 & -.171 & 301 & .864 & -0.02 & .12 & -.27 & .22 \\
\hline & No & & & -.171 & 300.329 & .864 & -0.02 & .12 & -.27 & .22 \\
\hline \multirow{2}{*}{$\begin{array}{l}\text { Para mi familia es muy } \\
\text { importante triunfar en la } \\
\text { vida. }\end{array}$} & Sí & 0.008 & .93 & 1.424 & 301 & .156 & 0.13 & .09 & -.05 & .32 \\
\hline & No & & & 1.423 & 300.225 & .156 & 0.13 & .09 & -.05 & .32 \\
\hline \multirow{2}{*}{$\begin{array}{l}\text { En mi casa somos muy } \\
\text { ordenados y limpios. }\end{array}$} & Sí & 5.793 & .02 & 2.572 & 301 & .011 & 0.31 & .12 & .07 & .54 \\
\hline & No & & & 2.567 & 294.370 & .011 & 0.31 & .12 & .07 & .54 \\
\hline \multirow{2}{*}{$\begin{array}{l}\text { Todos nos esforzamos } \\
\text { mucho en lo que hacemos } \\
\text { en casa. }\end{array}$} & Sí & 2.113 & .15 & .963 & 301 & .336 & 0.10 & .11 & -.11 & .32 \\
\hline & No & & & .961 & 295.702 & .337 & 0.10 & .11 & -.11 & .32 \\
\hline \multirow{2}{*}{$\begin{array}{l}\text { En mi familia es muy } \\
\text { importante aprender algo } \\
\text { nuevo o diferente }\end{array}$} & Sí & 1.162 & .28 & 1.723 & 301 & .086 & 0.19 & .11 & -.03 & .40 \\
\hline & No & & & 1.721 & 297.098 & .086 & 0.19 & .11 & -.03 & .40 \\
\hline \multirow{2}{*}{$\begin{array}{l}\text { Los miembros de la familia } \\
\text { guardan, a menudo, } \\
\text { sentimientos para sí mismos. }\end{array}$} & Sí & 1.419 & .24 & -.625 & 301 & .533 & -0.07 & .12 & -.31 & .16 \\
\hline & No & & & -.624 & 296.435 & .533 & -0.07 & .12 & -.31 & .16 \\
\hline \multirow{2}{*}{$\begin{array}{l}\text { En nuestra familia a veces } \\
\text { discutimos. }\end{array}$} & Sí & 0.772 & .38 & -2.370 & 301 & .018 & -0.30 & .13 & -.55 & -.05 \\
\hline & No & & & -2.372 & 300.998 & .018 & -0.30 & .13 & -.55 & -.05 \\
\hline \multirow{2}{*}{$\begin{array}{l}\text { En general ningún miembro } \\
\text { de la familia decide por su } \\
\text { cuenta. }\end{array}$} & Sí & 0.046 & .83 & .057 & 301 & .954 & 0.01 & .12 & -.23 & .25 \\
\hline & No & & & .057 & 300.915 & .954 & 0.01 & .12 & -.23 & .25 \\
\hline \multirow{2}{*}{$\begin{array}{l}\text { En casa solo estamos } \\
\text { pasando el rato. }\end{array}$} & Sí & 1.381 & .24 & -.271 & 301 & .787 & -0.03 & .13 & -.28 & .21 \\
\hline & No & & & -.270 & 297.296 & .787 & -0.03 & .13 & -.28 & .21 \\
\hline \multirow{2}{*}{$\begin{array}{l}\text { Cuando me quejo de algo } \\
\text { que me molesta se enojan } \\
\text { en mi familia. }\end{array}$} & Sí & 1.458 & .23 & 1.015 & 301 & .311 & 0.19 & .19 & -.18 & .56 \\
\hline & No & & & 1.023 & 251.409 & .307 & 0.19 & .19 & -.18 & .56 \\
\hline
\end{tabular}


Tabla 6 (Continuación)

Prueba T de muestras independientes

\begin{tabular}{|c|c|c|c|c|c|c|c|c|c|c|}
\hline & \multirow{3}{*}{$\begin{array}{l}\text { Se han } \\
\text { asumido } \\
\text { varianzas } \\
\text { iguales }\end{array}$} & \multicolumn{2}{|c|}{$\begin{array}{c}\text { Prueba de } \\
\text { Levene }\end{array}$} & \multirow[b]{3}{*}{$t$} & \multirow[b]{3}{*}{ gl } & \multirow[b]{3}{*}{ Sig. } & \multirow{3}{*}{$\begin{array}{c}\begin{array}{c}\text { Prueba } \\
\text { T }\end{array} \\
\\
\text { Dif. de } \\
\text { Medias }\end{array}$} & \multirow[b]{3}{*}{ ET } & \multirow{2}{*}{\multicolumn{2}{|c|}{ IC 95\% }} \\
\hline & & \multirow[b]{2}{*}{$\mathrm{F}$} & \multirow[b]{2}{*}{ Sig. } & & & & & & & \\
\hline & & & & & & & & & LI & LS \\
\hline \multirow{2}{*}{$\begin{array}{l}\text { En mi casa a veces los } \\
\text { miembros de la familia } \\
\text { discutimos y nos enojamos } \\
\text { tanto que golpeamos o } \\
\text { rompemos cosas. }\end{array}$} & Sí & 4.763 & .03 & $\begin{array}{l}-1.583 \\
\end{array}$ & 301 & .115 & -0.22 & .14 & -.49 & .05 \\
\hline & No & & & $\begin{array}{l}-1.580 \\
-\end{array}$ & 294.642 & .115 & -0.22 & .14 & -.49 & .05 \\
\hline \multirow{2}{*}{$\begin{array}{l}\text { En mi casa una sola } \\
\text { persona toma la mayoría } \\
\text { de las decisiones. }\end{array}$} & Sí & 0.042 & .84 & -.124 & 301 & .902 & -0.02 & .14 & -.30 & .26 \\
\hline & No & & & -.124 & 300.775 & .902 & -0.02 & .14 & -.30 & .26 \\
\hline
\end{tabular}

cuidadosamente" y "En mi casa somos muy ordenados y limpios". Por otro lado, las mujeres presentan puntuaciones elevadas en "En nuestra familia a veces discutimos".

\section{DISCUSIÓN}

Encontramos que alrededor 2 de cada 10 estudiantes mostraron un nivel de no aprobación de sus estudios, siendo esto no deseable, asimismo de 3 de 10 estudiantes mostraron bajas calificaciones, lo cual nos hace ver el nivel de dificultad del fenómeno de estudios en nuestro contexto.

Los datos obtenidos permiten una comprensión inicial de la relación entre el rendimiento académico y el clima familiar. En este sentido las dimensiones que más se relacionan de forma negativa y significativa con el rendimiento académico son la interacción familiar negativa y la independencia familiar. A su vez la interacción familiar negativa se relaciona de forma positiva con la independencia y con socialización de la familia. Esto lo podemos interpretar en el sentido de que los estudiantes al tener malas relaciones con su familia puede incidir en su bajo rendimiento académico, al no sentirse apoyados o sentirse desmotivados a seguir adelante en sus estudios. A la vez, el sentirse independientes quizás eso haga que la libertad con la que cuentan no la empleen a cuestiones educativas sino a otros intereses. Así la socialización que tiene con la familia podría estar matizada por desinterés de las cuestiones educativas. Esto está en consonancia con lo que explican otros autores en cuanto a que el uso que se le da al tiempo libre producto de la independencia en los adolescentes se ha mostrado como un factor relevante en el rendimiento académico (Kerr y Stattin, 2000). Así, encontramos que los adolescentes con los promedios más bajos presentan un clima familiar negativo, asociado a poca comunicación entre los miembros de la familia y donde los padres suelen tener ideas y comportamientos autoritarios. Además, los integrantes de estas familias a menudo guardan sentimientos para sí mismos, suelen discutir, así como ningún miembro de la familia decide por su cuenta. Estos resultados están en concordancia con los obtenidos con otros estudios, en el ámbito de la adolescencia (Chuchón, 2018; Aguilar, 2015; Álvarez et al., 2015; Guerrero, 2014; Parra y Oliva, 2006).

De igual forma, podemos entender que la poca comunicación que se tiene en familias con un clima negativo no permita que los adolescentes desarrollen la suficiente confianza para poder hablar con sus padres respecto a las probables dificultades que 
puedan encontrar en sus estudios y por lo cual no obtener apoyo para la resolución de este tipo de problemas, aspectos que ya han sido señalados por otros autores (Parra y Oliva, 2006). Asimismo, una relación de comunicación basada en el autoritarismo puede inhibir la confianza de los estudiantes y provocar miedo o temor de hablar respecto a temas que consideren puedan irritar a sus padres. Este tipo de clima familiar también puede provocar no concentrarse en sus tareas y trabajos escolares o perder motivación para los estudios dadas las dificultades que se pueden ir incrementando cuando se van acumulando las materias reprobadas. Además, esto probablemente puede afectar a tener un buen desarrollo psicológico en los jóvenes. Este resultado es similar a lo encontrado en otros estudios (Hernando, Oliva y Pertegal, 2012), donde además se señala que el establecimiento de límites claros, pero no un nivel excesivo de control, permiten un desarrollo más saludable (Mazo, Mejía y Muñoz, 2019).

Respecto a las diferencias entre varones y mujeres en los indicadores del clima familiar, encontramos que los varones más que las mujeres refieren que las actividades familiares se planifican cuidadosamente y que la familia es ordenada y limpia. Esto lo podemos interpretar en el sentido de que a los hombres se les enfatizan más las reglas de casa y dentro de ello lo relativo al orden y la limpieza como parte de su rol de género. En el caso de las mujeres, ellas mencionan más que en la familia suelen presentarse conflictos en sus relaciones. Esto se puede explicar en el sentido que las mujeres son más sensibles a detectar las dificultades familiares o tienden a darle más importancia que los hombres a los problemas, quizás esto asociado al rol tradicional de género en cuanto a que las mujeres tienen que ver más hacia dentro de la familia, en cuanto a ver más por la misma, es decir, estar más al cuidado y atención, lo cual ya han referido otros estudios (Torres, 2011)

Contrario a lo que se han encontrado en otros estudios (Estévez, Murgui, Musitu y Moreno, 2008; Lastre, López y Alcázar, 2018), no se ha encontrado en el presente trabajo relaciones significativas entre un clima familiar positivo y rendimiento académico. Pero sí se ha hallado relaciones significativas entre clima familiar positivo y la religiosidad y la socialización de la familia. Esto nos habla, creemos, de que la variable religión juega un papel importante en la interacción familiar, lo cual no es de extrañar ya que de acuerdo al último censo en el país (INEGI, 2010) alrededor del $92 \%$ de los ciudadanos se consideraron religiosos. Asimismo es probable que la socialización que tenga la familia sea con personas de ideas afines. Asimismo, estudios previos realizados por los autores han mostrado que la motivación intrínseca más que el clima familiar y el clima social predicen de mejor forma el rendimiento académico (Barreto y Álvarez, 2017; 2020). En ese mismo sentido otros autores han señalado que las actividades académicas exigen el empleo de procesos cognitivos complejos, por lo cual las aptitudes y la inteligencia son también buenos predictores del rendimiento académico (Lamas, 2015).

Los resultados obtenidos en el presente trabajo tienen interesantes implicaciones para la intervención educativa de los adolescentes, donde se recomienda establecer programas orientados a los jóvenes y sus padres donde se enseñe el papel que juegan tanto de las relaciones familiares positivas como de las negativas en el rendimiento académico de los alumnos y cómo mejorar las mismas. Además, es importante trabajar con ellos estrategias de comunicación asertiva en la resolución de conflictos familiares.

Por último, se recomienda se sigan llevando a cabo en nuestro contexto estudios en nivel medio superior analizando las características y el comportamiento de los estudiantes, su entorno familiar y social y su relación con el rendimiento académico. Así mismo, se recomienda trabajar en investigaciones futuras donde se pueda observar no solo el rendimiento académico en función del clima familiar, sino también con otras variables como, hábitos de estudio, autoeficacia, resiliencia, motivación, estrés escolar, entre otras variables que pueden ser importantes para estudiar y explicar estos fenómenos. 
- Conflicto de intereses.

Los autores declaran no tener ningún conflicto de intereses.

\section{REFERENCIAS}

Aguilar, F. (2015). Estudio comparativo del Clima Social Familiar en estudiantes de secundaria según su rendimiento académico de la Institución Educativa No 1279 Huaycán, Zona R. Lima: Universidad Peruana Unión.

Álvarez A., Suárez, N., Tuero, E., Núñez, J.C., Valle, A., y Regueiro, B. (2015). Implicación familiar, autoconcepto del adolescente y rendimiento académico. European Journal of Investigation in Health, Psychology and Education, 5(3), 293-311. doi:10.30552/ ejihpe.v5i3.133

Barreto, F. (2017). Motivación de logro, hábitos de estudio, clima escolar y clima familiar como predictores del rendimiento académico en estudiantes de bachillerato. Tesis de grado. Facultad de Psicología, UANL.

Barreto, F.J., y Álvarez, J. (2017). Clima Escolar y Rendimiento Académico en Estudiantes de preparatoria. Daena: International Journalof Good Conscience, 12(2), 31 -44.

Barreto, F.J., y Álvarez, J. (2020). Las dimensiones de la motivación de logro y su influencia en rendimiento académico de estudiantes de preparatoria. Enseñanza e Investigación en Psicología, 1 (3), 73-83.

Bronfenbrenner, U. (1992). Ecological systems theory. En R. Vasta (Ed.), Six theories of child development: Revised formulations and current issues (pp. 187-249). Jessica Kingsley Publishers.

Bronfenbrenner, U. (2004). Making human beings human. Thousand Oaks, CA: Sage

Buote, C. (2000). Relations of autonomy and relatedness to school functioning and psychological adjustment during adolescence $(T)$. Retrospective Theses and Dissertations, 1919-2007. Tomado desde https://open.library.ubc.ca/collections/ ubctheses/831/items/1.0053902

Caplan, S., Henderson, C., Henderson, J., \& Fleming, S. (2002). Socioemotional factor contributing to adjustment among early-entrance college students. Gifted Child Quarterly, 46(2), 124-134. doi:10.1177/001698620204600205
Castro, L. (2015). Influencia del clima social familiar en el rendimiento académico de los alumnos del $3^{\circ}, 4^{\circ}$ y $5^{\circ}$ grado del nivel secundario de la I.E. Divino Maestro N'80016. Paragueda, Otuzco. Trujillo: Universidad Nacional de Trujillo.

Chao, R. (2001). Extending Research on the Consequences of Parenting Style for Chinese Americans and European Americans. Child Development, 72(6), 1832-1843. doi:10.1111/1467-8624.00381

Chong, M. (2015). Clima social familiar y asertividad en alumnos secundarios del distrito de la Esperanza, Trujillo. Tesis de Pregrado. Trujillo: Universidad Privada Antenor Orrego.

Chuchón, J. (2018). Clima social familiar y rendimiento académico en los estudiantes de la I.E.P. Divina Providencia de Chincha. Lima: Universidad Inca Garcilaso De La Vega.

Claro, S. (2013). Calidad en educación y clima escolar: apuntes generales. Estudios Pedagógicos, 39, 347-359. doi:10.4067/ S0718-07052013000100020

Coleman, J. (1966). Equality of educational opportunity. Washington, DC: Government Printing Office.

Estévez, E., Murgui, S., Musitu, G., y Moreno, D. (2008). Clima familiar, clima escolar y satisfacción con la vida en adolescentes. Revista Mexicana de Psicología, 25(1), 119 128.

Fernández-Ballesteros, R., y Sierra, B. (1984). Escalas de clima social: familia, trabajo, instituciones penitenciarias, centro escolar. Madrid: Tea Ediciones, S.A.

Garg, R., Levin, E., Urajnik, D., \& Kauppi, C. (2005). Parenting style and academic achievement for East Indian and Canadian adolescents. Journal of Comparative Family Studies, 36, 653-661.

García-Lastra, M. (2013). Educar en la sociedad contemporánea. Hacia un nuevo escenario educativo. Convergencia. Revista de Ciencias Sociales, 20(62), 199-220.

Guerrero, Y. (2014). Clima social familiar, inteligencia emocional y rendimiento académico de los alumnos de quinto de secundaria de las instituciones educativas públicas de Ventanilla. Lima: Universidad Nacional Mayor de San Marcos. 
Hernando, A., Oliva, A., y Pertegal, M.A. (2012). Variables familiares y rendimiento académico en la adolescencia. Estudios de Psicología, 33(1), 51 -65.

INEGI (2010). Panorama de las religiones en México 2010. Aguascalientes: Instituto Nacional de Estadística y Geografía.

Instituto Nacional para la Evaluación de la Educación (2019). La educación obligatoria en México Informe 2019. Ciudad de México: INEE.

Kerr, M., y Stattin, H. (2000). What parents know, how they know it, and several sorms of adolescent adjustment: Further support for a reinterpretation of monitoring. Developmental Psychology, 36, 366-380.

Lamas, H. (2015). Sobre el rendimiento escolar. Propósitos y Representaciones, 3(1), 313386. doi:10.20511/pyr2015.v3n1.74.

Lastre, K., López, L., y Alcázar, C. (2018). Relación entre apoyo familiar y el rendimiento académico en estudiantes colombianos de educación primaria. Psicogente, 21 (39), 102-1 15. doi:10.17081/psico.21.39.2825

Marchesi, A., y Martín, E. (2002). Evaluación de la educación secundaria. Fotografía de una etapa polémica. Madrid: CESMA.

Mazo, Y. I., Mejía, L. A., y Muñoz, Y. P. (2019). Calidad de vida: la familia como una posibilidad transformadora. Revista Poiésis, 36, 98-110. doi:10.21501/16920945.3192

Martínez-Pampliega, A., Merino, L., Iriarte, L., y Olson, D.H. (2017). Psychometric Properties of the Spanish version of the Family Adaptability and Cohesion Evaluation Scale IV. Psicothema, 29(3), 414-420.

Moos, R., Moos B., y Trickett, E. (1984). FES, WES y CES. Escalas de clima social. Madrid, España: TEA Ediciones.

Morales, L., Morales, V., y Holguín, S. (2016). Rendimiento escolar. Humanidades, Tecnología y Ciencia, 15, 1-5.

Moreno, D., Estévez, E., Murgui, S., y Musitu, G. (2009). Relación entre el clima familiar y el clima escolar: el rol de la empatía, la actitud hacia la autoridad y la conducta violenta en la adolescencia. International Journal of Psychology and Psychological Therapy, 9(1), 123- 136.

Núñez, A., (2009). Participación de padres de familia en la educación de sus hijos en nivel secundaria. México: Instituto Tecnológico de Sonora.

Oliva, A., Parra, A., y Arranz, E. (2008). Estilos relacionales parentales y ajuste adolescente. Infancia y Aprendizaje, 31(1), 93-106. doi: $10.1174 / 021037008783487093$

Oliva, E., y Villa, V.J. (2014). Hacia un concepto interdisciplinario de la familia en la globalización. Justicia Juris, 10(1), 11 20.

Olson, D.H, McCubbin, H.I., Barnes, H., Larsen, A., Muxen, M., y Wilson, M. (1989). Families: What Makes Them Work. Beverley Hills, USA: SAGE Publications, Inc.

Parra, A., y Oliva, A. (2006). Un análisis longitudinal sobre las dimensiones relevantes del estilo parental durante la adolescencia. Infancia y Aprendizaje, 29, 453-470.

Pelegrina, S., García, M., y Casanova, P. (2002). Los estilos educativos de los padres y la competencia académica de los adolescentes. Infancia y aprendizaje, 25(2), 147-168. doi:10.1174/021037002317417796

Pittman, L., \& Chase-Lansdale, P. (2001). African American adolescent girls in impoverished communities: Parenting style and adolescent outcomes. Journal Research Adolescence, 11, 199-224. doi:10.1111/1532-7795.00010

Rodríguez. P. (201 1). Análisis de la convivencia escolar en aulas de Educación Primaria. Revista Iberoamericana de Educación, 55(3), 1-12. doi:10.35362/rie5531599

Saconne, M. (2017). La obligatoriedad de la Educación Media Superior. Apuntes para pensar la experiencia mexicana. Teorías y procesos educativos, 13, 122-139. doi:10.19137/an 1309

Sánchez, P., y Valdés, A. (2011). Una aproximación a la relación entre el rendimiento académico y la dinámica y estructura familiar en estudiantes de primaria. Revista Intercontinental de Psicología y Educación, 13, 177-196.

Solano, L. (2015). Rendimiento académico de los estudiantes de secundaria obligatoria y su relación con las aptitudes mentales y las actitudes ante el estudio (Tesis de doctorado). Madrid: UNED.

Solís, F., y Aguiar, R. (2017). Análisis del papel del involucramiento de la familia en la 
escuela secundaria y su repercusión en el rendimiento académico. Sinéctica, 49.

Suárez, N., Fernández, E., Cerezo, R., Rodríguez, C., Rosário, P., y Núñez, J. (2012). Tareas para casa, implicación familiar y rendimiento académico. Aula Abierta, 40(1), 73-84.

Torres, L. (2011). Rendimiento académico, familia y equidad de género. Ciencia y Sociedad, 36(1), 46-64.
Verdugo, J., Arguelles, J., Guzmán, J., Márquez, C., Montes, R., y Uribe, I. (2014). Influencia del clima familiar en el proceso de adaptación social del adolescente. Psicología desde el Caribe, 31 (2), 207-222. 